\title{
PULSED-NEUTRON-SOURCE MODELS FOR THE ASTROPHYSICAL S-PROCESS
}

\author{
K. M. Howard, G. J. Mathews, K. Takahashi, \\ and R. A. Ward \\ Un iversity of California \\ Lanrence Livermore National Laboratory \\ Liverwore, CA 94550
}
This paper was prepared for subnittal to
the Fifth Moriond Astrophysics Meeting
Les Arcs, France
March 17-23, 1985

May 1985

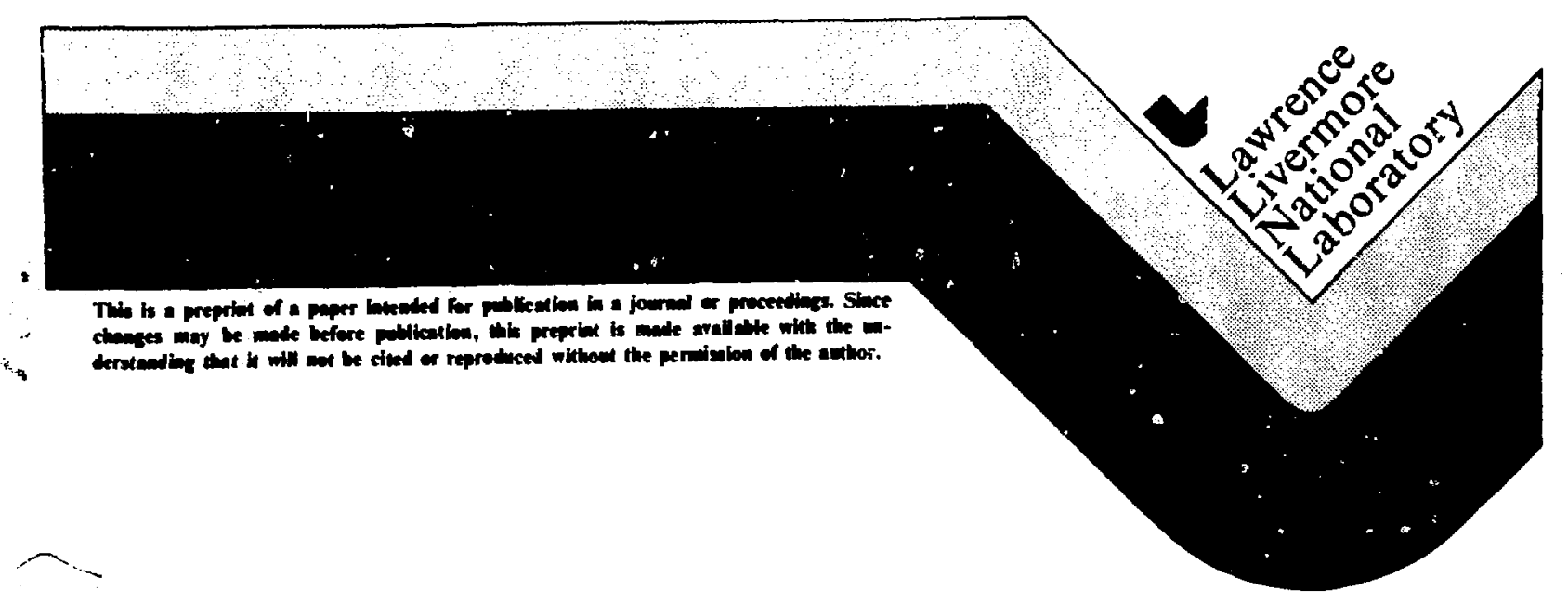


ABSTRACT. The astrophysical s-process is a sequence of neutron-capture and beta-decay reactions on a low time scale compared to beta-decay lifetimes near the line of stability. We systematically $s$ tudy this detailed sequence of neutron cspture, continuum and bound-state beta decay, positron decay, and electron-capture reactions that comprise the s-process for a broad range of astrophysical environments. Our results are then compared with the solar-system abundances of heavy elements to determine the range of physical conditions responsible for their nucleosynthesis.

\section{INTRODUCTION}

It has been clear for some time ${ }^{\bar{i}}$ that an exponential distribution of neutron exposures is required to fit the solar-system s-process $\sigma \mathrm{N}$ curve (neutron capture cross section times abundance) as a function of atomic mass. Ul rich 2,3 showed that an exponential distribution of exposures could be achieved in a single star which subjected initial seed material to periodic neutron exposures followed by dredge up of some fraction of the irradiated material to the stellar surface. This s-process scenario has been explored in a series of papers ${ }^{4-7}$ based on a $22 \mathrm{Ne}(\alpha, n)^{25} \mathrm{Mg}$ neutron source for the s-process during thermal pulses of asymptotic giant branch stars. There is sufficient uncertainty in the stellar models, however, that a different approach is warranted, i.e. to utilize the observed solar-system s-process $\mathrm{NN}$ curve to define the constraints on any stellar model for the s-process. This is the subject of the present work. This study reveals that the s-process is best fit with conditions similar to those expected for relatively low-mass red-giant stars near the end of their 1 ifetime. Deviations of the fit from the observed solar-system values highlights the need for improved nuclear data.

\section{S-PROCESS CALCULATION}

In the classical s-process (without beta-decay branching) the abundance of an isotope is given simply by the solution to the set of

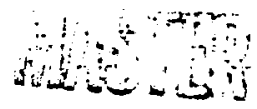


coupled differential equations,

$$
d N_{A} / d \tau=\sigma(A-1) N_{A-1}-\sigma(A) N_{A},
$$

where $\tau$ is the tine integrated neutron flux (i.e. neutron exposure) and $\sigma$ the Maxwellian averaged neutron capture cross section.

at equilibrium, a single exposure would lead to a constant oN value for 11 isotopes in the s-process. The solar-system on curve (see Fig. 1), however, requires an exponential distribution of neutron exposures, i.e. the probability, $\rho(\tau)$ for a given exposure is taken to be,

$$
\rho(\tau) \propto \exp \left(-\tau / \tau_{0}\right)
$$

In a periodic s-process operating in a single star ${ }^{2}, 3$, the mean exposure, $\tau_{0}$, is simply related to the average exposure per pulse, $\Delta \tau$, and the fraction of material, $r$, which remains after each dredge up, i.e. $\tau_{0}=-\Delta \tau / 1 n(r)$. Note, that the mixing fraction and exposure per pulse are not independent parameters.

In dynamic stellar environments, such as thermally-pulsing red giants, the simplicity of the classical s-process (Eq. 1) is lost due to a break down of the assumption that neutron captures are slow compared to beta decay. We therefore, compute the nucleosynthesis in a network:

$$
\begin{aligned}
\frac{d N(Z, A)}{d t}= & N(Z, A-1) n_{n} \sigma_{n, \gamma}(Z, A-1)+N(Z-1, A) \lambda_{B}(Z-1, A) \\
& -N(Z, A)\left[n_{n} \sigma_{n, \gamma}(Z, A)+\lambda_{B}(Z, A)\right] .
\end{aligned}
$$

The time-dependence of the flux and temperature dependence of the cross sections and beta rates are included. In a few cases, positron, electron-capture, or alpha decay must also be added to Eq. (3). We utilize experimental neutron-capture cross sections when available $8-16$. Cross sections for unstable or unmeasured nuclei wer $\leqslant$ taken from Hauser-Feshbach estimates 17 along with the temperature dependence of all cross sections. Decay rates from thermally populated excited states were calculated assuming thermal equilibrium and with appropriately choosen $\mathrm{ft}$ values 18. The initial seed abundances were taken from solar-system values ${ }^{19}$.

\section{RESULTS}

We have adjusted the parameters to minimize $\chi^{2}$ for the fit to 23 s-only nuclei with $Z \geq 40$. Lighter nuclei were onitted because of possible contribution from other sources 9 . The nost sensitive parameters in the fit are $\tau_{0}$, and $n_{n}$, although the temperature, pulse shepe, and interpulse period also enter.

Figure 1 is an example of a good fit $\left(y^{2}{ }_{2} .3\right)$ for a mean exposure of $\tau_{0}=0.277 \mathrm{mb}^{-1}$, a constant density $\left(n_{n}=\right.$ $\left.1.0 \times 10^{8} \mathrm{~cm}^{-3}\right)$ and temperature $\left(T=0.348 \times 10^{9} \mathrm{~K}\right)$, with a long 


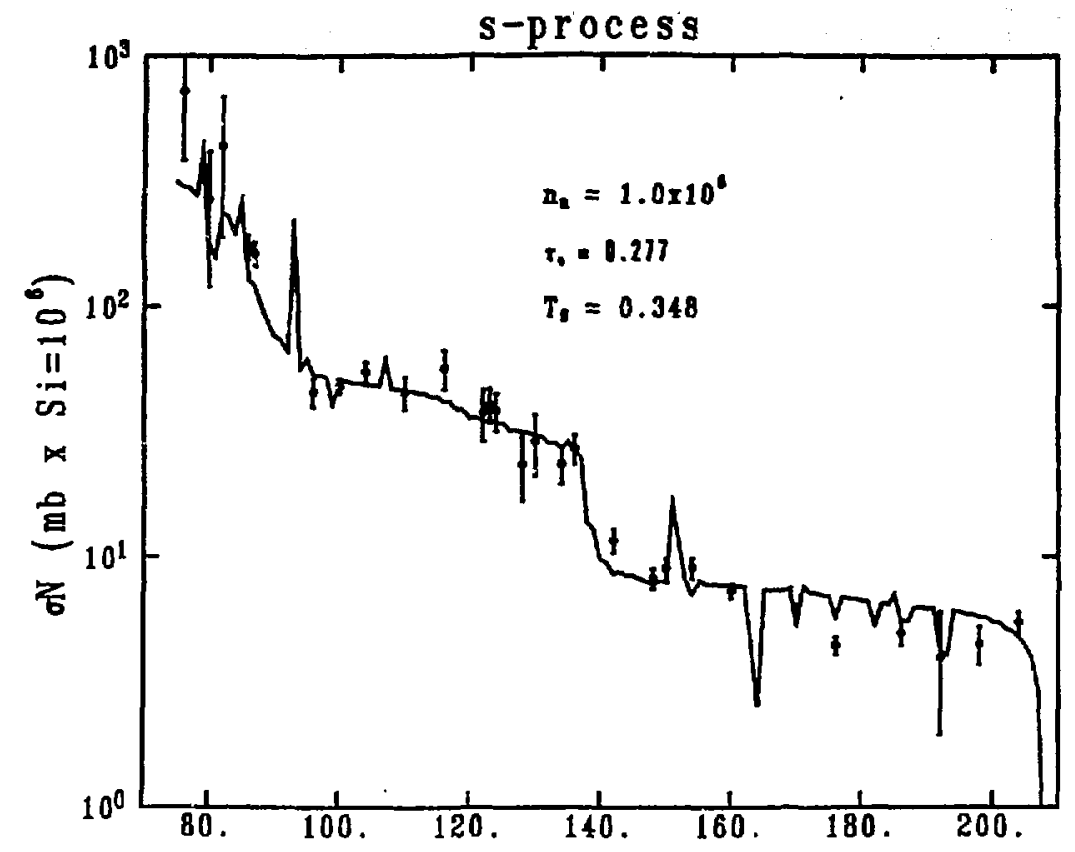

A

Figure 1 Fit to the observed oN curve.

interpulse interval ( $2460 \mathrm{y}$ ). The $x^{2}$ quickly increases for higher fluxes, but increases more slowly as the flux is decreased. From this study we conclude that the best-fit parameters are $n_{n}=$ $\left.1.0\left( \pm 8: \frac{6}{2}\right) \times 10^{8} \mathrm{~cm}^{-3}, \mathrm{~T}=0.30( \pm 0.04) \times 10^{9} \mathrm{~K}\right)$, and

$\tau_{a}=0.28 \mathrm{~s} 0.01\left(\mathrm{mb}^{-1}\right)$, corresponding to a pulse duration oq $500\{ \pm 108$ ) yrs. This is consistent with previous estimates 3,14 of the neutron density based on one or two branches alone. For higher densities than $10^{8} \mathrm{~cm}^{-3}$ many branches (al1 above $A=140$ ) are bypassed, while for low densities the oN curve approximates the nominal s-process. What is most interesting is that these stellar parameters, (derived solely from input nuclear data and observed abundances) are very similar to the conditions thought to exist $t^{4}$ in relatively low-mass (M 5 $3 \mathrm{M}_{0}$ ) red-giant stars as they ascend the asymptotic giant branch. The best fit for the solar-systen $\sigma \mathrm{N}$ curve exhibits pronounced deviations from the smooth monotonically decreasing classical s-process curve. The reason for this is that many stable nuclei are actually produced as beta-unstable progenitors due to neutron captures on beta-unstable nuclei. If the progenitor has a larger (or smaller) cross section there will be a dip (or peak) in the oN curve when $\sigma N$ for the stable daughter is calculated. 
This is the reason for the pronounced peaks on Fig. 1 at ${ }^{93} \mathrm{Nb}$ (partially pioduced as $93_{\mathrm{Zr}}$ ) and $15 \mathrm{l}_{\mathrm{Eu}}$ (partially produced as $151_{\text {sm). }}$.

For a broed renge of everage neutron densities the temperature minim occure for $T_{9}=0.30( \pm 0.04)$ ( $\mathrm{kT}=26 \pm 4 \mathrm{keV}$ ). This is due both to the change in cross sections and the fact that certain beta decaya are too slow at low temperature to provide adequate competition with neutron capture at certain key branch points (e.g. $79 \mathrm{se}, 134 \mathrm{Cs}$, and $154 \mathrm{Gd}$ ). at low temperatures the fact that $8^{80} \mathrm{Kr},{ }^{134} \mathrm{Ba}$, and ${ }^{154} \mathrm{Gd}$ are bypassed is particularly evident. The fact that $154 \mathrm{Gd}$ is bypased is at first surprizing since the precursor is ${ }^{154} \mathrm{Eu}$ with a terrestrial half 1 ife of only 8 yrs. The capture cross section for ${ }^{154} \mathrm{Eu}$ is extremely large ( 8 barns at $k T=$ $10 \mathrm{keV}$ ), however, so that capture occurs at these neutron densities before bete decay. The poorer $f$ it at high temperature is due primarily to changes in the neutron capture cross sections; also the increased beta-rates at high temperatures tends to washout the branches at $170 \mathrm{Yb}$ and $1860 \mathrm{~s}$. Thus, we find that the best solution is for $\mathrm{kT}=26 \mathrm{keV}, \mathrm{n}_{\mathrm{n}}=10^{8} \mathrm{~cm}^{-3}$, and $\tau_{0}=0.28 \mathrm{mb}^{-1}$. The rather narrow range of allowed temperatures for a broad range of densities is significant because these temperatures are characteristic of the $22 \mathrm{Ne}(\alpha, n)^{25} \mathrm{Mg}$ source reaction. Temperatures in this range are just what is required to give the correct neutron exposure from this reaction. Most other possible s-process sources such as $13_{\mathrm{C}}(a, n) 16_{0}$ and carbon burning would normally be expected to occur at temperatures considerably lower or higher than the ranges which give the best fit to the oN curve. It is also encouraging that this completely independent means of estimating the temperature (which makes no assumption about the stellar site other than that it experiences sequential neutron irradiations) comes so close to the traditionally assumed value for $k T=30 \mathrm{keV}$ based on the assumption that this process should occur at typical helium-burning temperatures.

\section{ACKNOWLEDGEMENT}

Work performed under the auspices of the U.S. Department of Energy by the Lawrence Livermore National Laboratory under contract number W-7405-ENG-48.

\section{REFERENCES}

1. P. A. Seeger, W. A. Fowler, D. D. Clayton, Astrophys. J. Suppl., 97, 121 (1965).

2. $\overline{R . ~ K . ~ U l r i c h, ~ i n ~ " E x p l o s i v e ~ N u c l e o s y n t h e s i s ", ~ e d s . ~ D . ~ N . ~ S c h r a m m ~}$ and W. D. Arnet (Univ. Texas Press, Austín, 1973) p.139.

3. R. K. Ulrich, in "Essays in Nuclear Astrophysics", eds. C. A. Barnes, D. D. Clayton, and D. N. Schramm (Cambridge Univ. Press, N. Y., 1982) P. 301.

4. I. Iben, Jr., Ap. J., 217, 788 (1977).

5. J. W. Truran and I. Iben, Jr., Ap. J., 216, 197 (1977).

6. I. Iben, Jr., and J. W. Truran, Ap. J., 220, 980 (1978). 
7. K. R. Cosner, I. Iben, and J. R. Truran, Ap. J. Lett., 238, L91 (1980).

8. B. J. Allen, J. H. Gibbons, and R. L. Mackl in, Adv. Mucl. Phy., 4, 205 (1971).

9. F. Kuppeler, H. Beer, K. Wisshak, D. D. Clayton, R. L., Macklin, and R. A. Ward, Ap. J., 257, 821 (1982).

10. M. J. Newman, Ap. J., $21 \overline{9}, 676$ (1978).

11. H. Beer and R. L. Macklin, Phys. Rev., C26, 1404 (1982).

12. H. Seer, F. Kuppeler, G. Reffo, and G. Ventorini, Ap. Space Sci., 97,95 (1983).

13. $\bar{R}$. K. Winters, F. Kdippeler, K. Wisshak, A. Mengoni, and G. Reffo, (submitted to Ap. J., 1984).

14. H. Beer, F. Käppeler, K. Yokoi, and K. Takahashi Ap. J., 278, 388 (1984).

15. H. Beer and G. Walter, Astron. Ap. (1984 in press).

16. G. J. Mathews and F. Kippeler, Ap. J., 286, 810 (1984).

17. J. A. Holmes, S. E. Woosley, W. A., Fowier, end B. A. 2 immermen, Atom. Nuc1. Data Tables, 18, 306 (1976).

18. K. Takahashi and $K$. Yokoi (to be published).

19. E. Anders and M. Ebihara, Ceochim. Cosmochim. Acta, 46, 2263 (1982).

\section{DISCLAIMER}

This report was prepared as an account of work sponsored by an asency of the United States Government. Neither the United Stales Gowernment nor any agency theroof, nor any of their employees, makes any warranty, express or implied, or assumes any lezal liability or reaponsibility for the accuracy, completeness, or usefulness of any information, apparatus, product, or process disclowod, or represents that its use would not infringe privately owned rights. Reference herein to any upecific commercial product, process, or service by trade name, trademart, manufacturer, or otherwise does not necesurily constitute or imply its endorsemenh, recommendation, of favoring by the United Stales Government of any asency theroof. The view and opinions of authors expresed herein do not necesserily state or reflect thowe of the United Stuts Governmert or any asency thereof. 